\title{
Review
}

Journal of Innate

Immunity
J Innate Immun 2011;3:374-382

DOI: $\underline{10.1159 / 000323936}$
Received: October 29, 2010

Accepted after revision: December 24, 2010

Published online: March 12, 2011

\section{Modulation of Natural Killer Cell Anti-Tumor Reactivity by Platelets}

\author{
Theresa Placke Hans-Georg Kopp Helmut Rainer Salih \\ Department of Hematology/Oncology, Eberhard Karls University, Tübingen, Germany
}

\section{Key Words}

Natural killer cells $\cdot$ Platelets $\cdot$ Cancer $\cdot$ Metastasis

\begin{abstract}
Natural killer (NK) cells may prevent tumor progression and metastasis. Apart from the direct interaction with their targets, NK cell activity is influenced by the reciprocal interplay with other hematopoietic cells. While the interaction of NK cells e.g. with dendritic cells or monocytes/macrophages is well characterized, knowledge regarding their crosstalk with platelets, another central component of the blood, is still fragmentary. However, studies in mice and men clearly document a strong dependence of tumor progression and metastasis on quantitatively and functionally normal platelets. In mice, metastasis is inhibited by thrombocytopenia, and this effect is reversed by additional NK cell depletion, indicating that platelets may 'indirectly' contribute to tumor dissemination by impairing NK cell anti-tumor reactivity. In humans, circumstantial evidence indicates that metastasizing malignant cells do not travel through the blood alone, but efficiently attract and get coated by platelets, thereby causing release of platelet granule content. Beyond this secretion of various growth factors and cytokines/chemokines, platelets may also influence NK cell function by immunoregula-
\end{abstract}

tory molecules expressed on the platelet surface. Here, we review the available data regarding tumor-platelet-NK cell interaction focusing on metastatic tumor spread and discuss the molecular mechanisms underlying this trilateral crosstalk.

Copyright $\odot 2011$ S. Karger AG, Basel

\section{Introduction}

Natural killer (NK) cells are cytotoxic lymphocytes which play a major role in anti-tumor immunity and can prevent tumor progression and metastasis. The mechanisms leading to NK cell activation are described by the principles of 'missing self' and 'induced self', which imply that cells with low or absent expression of major histocompatibility complex (MHC) class I ('missing self') and/ or expression of stress-induced ligands for activating NK cell receptors ('induced self') are preferentially recognized and eliminated by NK cells. Beyond 'classical' NK cell receptors like killer immunoglobulin-like receptors

T.P., H.-G.K. and H.R.S. contributed equally to this work.

\section{KARGER}

Fax +4161306 1234

E-Mail karger@karger.ch

www.karger.com
(C) 2011 S. Karger AG, Basel

1662-811X/11/0034-0374\$38.00/0

Accessible online at:

www.karger.com/jin
Prof. Dr. med. Helmut R. Salih

Department of Hematology/Oncology, Eberhard Karls University Otfried-Mueller-Strasse 10

DE-72076 Tübingen (Germany)

Tel. +49 7071298 3275, E-Mail Helmut.Salih@med.uni-tuebingen.de 
(KIR) and natural cytotoxicity receptors, various other immunoregulatory molecules like NKG2D, Fc $\gamma$ RIII (CD16), members of the tumor necrosis factor (TNF) receptor family and many more influence NK cell reactivity [1]. Thus, a balance of activating and inhibitory signals mediated by a sophisticated repertoire of receptors determines the outcome of NK cell-tumor cell interaction.

Besides the direct interaction with their target cells, NK cell reactivity is further influenced by reciprocal interaction with other hematopoietic cells like dendritic cells, monocytes/macrophages or T cells [for an excellent review, see ref. 2]. However, little is known regarding the influence of platelets (a central component of the hemostatic system), which are physiologically in direct proximity of NK cells in the blood. This paucity of data regarding NK cell-platelet interaction is surprising since both cell types have been known to play a role in tumor progression for many years. Already in 1878, Billroth [3] identified viable neoplastic cells in blood clots and introduced the concept that the hemostatic system plays a pathogenetic role in tumor metastasis. Meanwhile, several studies have demonstrated that elevated platelet counts of tumor patients are associated with advanced cancer stage and represent a negative prognostic marker in several malignant diseases [4]. The first experimental evidence for the involvement of platelets in tumor progression was provided in 1968 by studies of Gasic et al. [5]. These investigators utilized a mouse model where syngeneic tumor cells injected into the tail vein get caught in the pulmonary microvasculature giving rise to metastases, which can be counted as nodules after 2-3 weeks, and reported that thrombocytopenia inhibits metastasis in a platelet count-dependent manner [5]. About 30 years later, Nieswandt et al. [6] used the very same model system and showed in an elegant set of experiments that concomitant depletion of NK cells reverses the anti-metastatic phenotype of thrombocytopenia.

Since then, platelets can no longer be regarded as innocent bystanders' when it comes to immune evasion of tumor cells. However, the molecular mechanisms underlying platelet-tumor-immune interaction and also modulation of NK cell reactivity by platelets are still incompletely understood. In theory, platelets may influence tumor progression through multiple mechanisms. (1) Interaction of platelets and tumor cells causes tumor cellinduced platelet aggregation and activation [7], upon which platelets release their granule contents. These comprise various growth factors like platelet-derived growth factor (PDGF) or vascular endothelial growth factor (VEGF) that may stimulate tumor proliferation and neo- angiogenesis. (2) Upon intravasation of metastasizing cells, platelets adhere to the cancer cell surface causing formation of tumor-platelet thrombi, which get caught in the microvasculature [8]. This may facilitate interaction with endothelial cells and penetration of the vessel wall opening the door to a metastatic niche $[8,9]$. (3) Platelets may influence reactivity of immune effector cells both by the multiple soluble factors secreted upon tumor cellplatelet interaction and by immunoregulatory molecules expressed on the platelet membrane. Data on the third mechanism with regard to modulation of NK cell reactivity are discussed in this article. As evidence for speciesspecific differences with regard to anti-tumor immune responses in mice and men is increasing, we emphasized the distinction between data obtained from murine models and the human system.

\section{Tumor-Platelet-NK Cell Interaction in the Murine System}

As stated above, already in 1968, Gasic et al. [5] showed that platelet depletion reduced the number of pulmonary metastases after tail vein injection of tumor cells in mice. Later work by Gasic et al. [7] and Pearlstein et al. [10] documented that tumor cells aggregate platelets in vitro and that there is a correlation between tumor cell-induced platelet aggregation by a given tumor cell line and its dependence on platelets when it comes to the formation of pulmonary metastases. Interestingly, the transfer of human platelets to thrombocytopenic mice that, as described above, were protected against metastasis restored metastasis to control levels [11]. Inhibition of platelet aggregation with aspirin, ticlopidine or prostacyclin $\left(\mathrm{PGI}_{2}\right)$ did not influence metastasis in the tail vein injection model $[11,12]$. In contrast, interference with platelet adhesion utilizing anti-von-Willebrand-factor antibody or anti-GpIIb/IIIa antibody inhibited metastasis and the metastasis-enhancing effect of platelet transfer in thrombocytopenic mice [11]. This indicated that adhesion may be more relevant than aggregation for enabling metastasis. Gorelik et al. [13] demonstrated anti-metastatic effects of heparin and $\mathrm{PGI}_{2}$ when wild-type mice received intravenous injections of B16 melanoma cells. The same investigators showed that concomitant depletion of NK cells was able to revert the anti-metastatic effects and were the first to hypothesize that anticoagulants may decrease metastasis by enabling NK cells to better target tumor cells rather than by inhibitory effects on tumor cell adhesion to the vascular wall. 


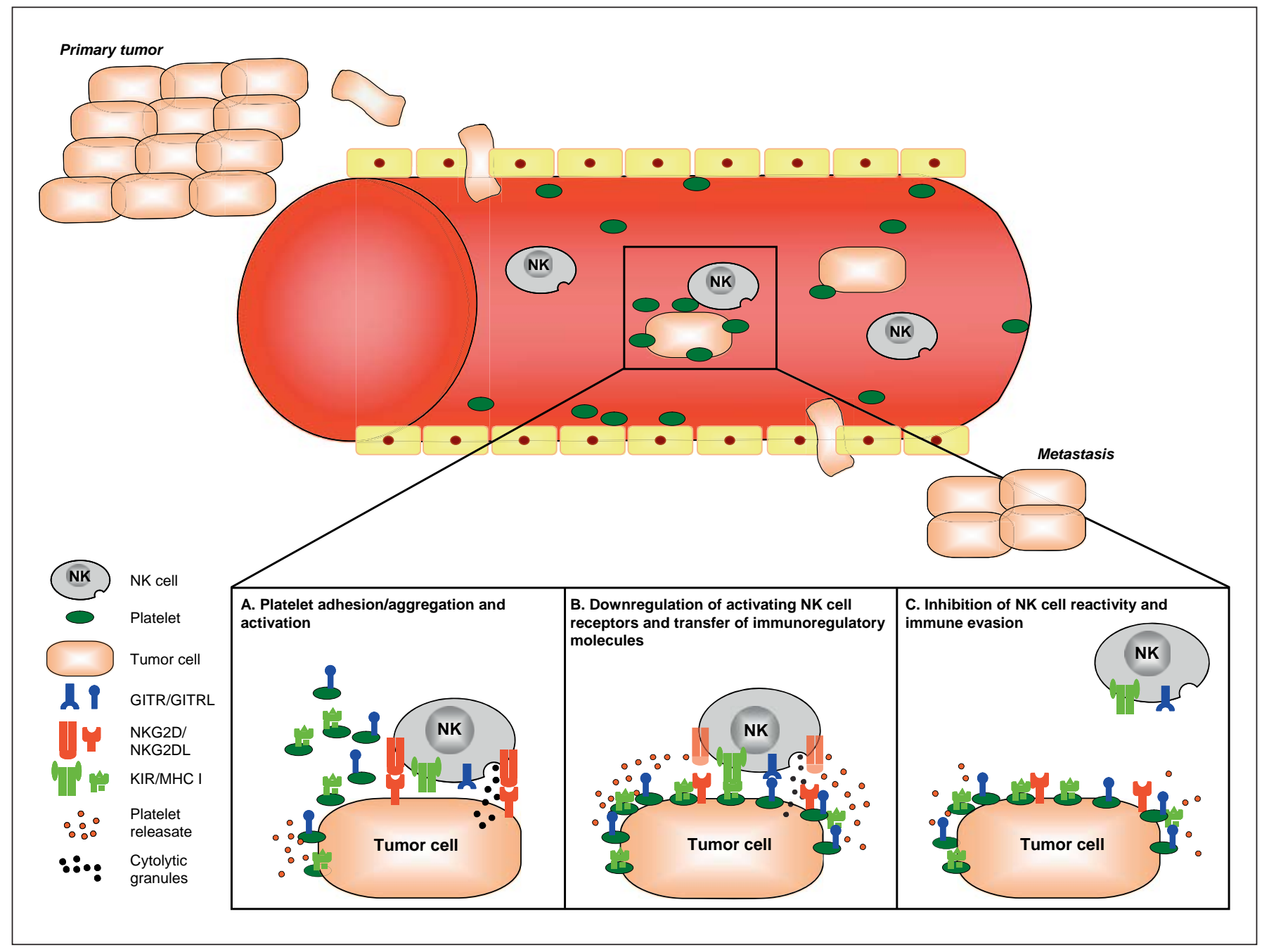

Fig. 1. Model of immunoinhibitory tumor-platelet-NK cell interaction in metastasis. Disseminating tumor cells enter the blood and rapidly get coated by platelets leading to platelet activation and degranulation (A). Platelet releasate impairs function of activating receptors on NK cells as exemplified by loss of NKG2D (B). Moreover, tumor cell coating confers expression of platelet-expressed immunoregulatory/immunoinhibitory molecules like MHC class I and GITRL to tumor cells. Reduced function of NK cell-activating receptors and 'pseudo expression' of immunoinhibitory molecules on platelet-coated tumor cells including 'pseudo self' impairs NK cell tumor immune surveillance by disturbing the 'induced self' and 'missing self' tumor cell recognition and elimination (C). Note that beyond the molecules employed for illustration, various other activating and inhibitory surface molecules and soluble factors are likely to contribute.
Of note, Jin et al. [14] demonstrated that platelets not only influence metastasis but also the growth of primary tumors in mice. Platelet and megakaryocyte counts in $\mathrm{Thpo}^{-/-}$(thrombopoietin deficient) and $\mathrm{Mpl}^{-/-}$(thrombopoietin receptor deficient) mice are reduced by $>90 \%$ as compared to wild-type levels [15]. In these $\mathrm{Thpo}^{-/-}$and $\mathrm{Mpl}^{-/-}$mice, the local tumor growth of transplanted syngeneic tumor cells [Lewis lung carcinoma (LLC), B16 melanoma, B6RV2 lymphoma and EL-4 thymoma] was significantly reduced. However, the protective effect of thrombocytopenia was more pronounced with regard to pulmonary and renal metastasis [14]. Interpretation of these data is facilitated when NK cells are introduced. In a mouse model of experimental metastasis, Nieswandt et al. [6] observed increased numbers of CFS1 lung metastases in $\mathrm{C} 3 \mathrm{H} / \mathrm{HeN}$ mice upon treatment with a monoclonal antibody to the interleukin (IL)- 2 receptor $\beta$-chain in order to deplete NK cells. Similar results were obtained 
with B16 melanoma cells, and NK cell depletion also resulted in an atypical metastatic pattern including liver metastasis. Of note, NK cell depletion did not influence liver involvement when NK cell-resistant ESb thymoma cells were injected. In this context it is of interest that $\mathrm{ESb}$ cells, in contrast to CFS1 and B16 cells, do not induce platelet aggregation in vitro. When the investigators employed mice after platelet depletion, they observed a $>95 \%$ reduced metastasis of CFS1 and B16 cells compared to control animals. This effect was reversed by additional depletion of NK cells, and in vitro analyses revealed that the presence of platelets reduced NK cell killing of YAC1 in a platelet count-dependent manner. Moreover, supernatants from both platelets alone and from platelets cocultured with YAC1 cells inhibited the natural cytotoxicity of murine splenocytes [6].

Several years later, Palumbo et al. [16] provided evidence that platelet activation may be crucial for their metastasis-facilitating effect. After intravenous injection of LLC or B16 cells, or spontaneously after xenotransplantation of LLC cells into the dorsal subcutis, Gaq-deficient $\left(\mathrm{G}_{\mathrm{aq}} \mathrm{q}^{-/}\right)$mice, which lack a $\mathrm{G}$ protein critical for platelet activation, developed less lung metastases while the qualitative features, size and overall appearance of metastases were comparable to control animals. Also, primary tumor growth was unaffected. Moreover, the lungs of $\mathrm{G} \mathrm{q}^{-1-}$ mice contained significantly less tumor cells $24 \mathrm{~h}$ after injection ( 1 vs. $10 \%$ of the LLC inoculum). Localization of tumor cells within the lungs and other tissues was similar in both groups. Of note, no decrease in pulmonary tumor cells was observed in ${\mathrm{G} \alpha q^{--}}^{-}$mice after NK cell depletion. These results indicate that mouse models with functional rather than quantitative platelet defects may serve to discriminate tumor-promoting effects of platelets with regard to NK cell inhibition and other NK cell-independent mechanisms. The same study also revealed an influence of the plasmatic hemostatic system on NK cells: fibrinogen-deficient mice had less pulmonary metastases and decreased pulmonary tumor cell survival after injection of LLC, and this effect was dependent on functionally intact NK cells [16].

In a subsequent study, the same investigators reported that tumor cell tissue factor expression dictates pulmonary metastasis upstream of coagulation-dependent mechanisms. Pulmonary metastasis in a mouse model was abrogated when tumor cells did not express tissue factor in mice with intact NK cell function. Of note, tissue factor expression remained an important determinant of metastasis even when NK cells were depleted, which led the investigators to postulate that tissue factor expression supports metastasis by both NK cell-dependent and -independent mechanisms. Moreover, Palumbo et al. [17] suggested that NK cell escape is fibrinogen dependent as well. Utilizing murine cells, Zheng et al. [18] confirmed in vitro that fibrinogen and thrombin facilitate the interaction of tumor cells and platelets as well as the inhibitory effects of platelets on NK cells. Palumbo et al. [19] also demonstrated a role for factor XIII in LLC and B16 pulmonary metastasis, which added further evidence for the involvement of the plasmatic coagulation system in NK cell immune evasion of tumor cells beyond the effect of platelets. Of note, none of the above mentioned genetic eliminations of molecules involved in hemostasis affected the cytolytic capacity of the NK cells.

In conclusion, murine metastasis models, albeit artificial in nature, clearly revealed a dependence of pulmonary metastasis on the presence of functioning platelets and an intact plasmatic coagulation system. These metastasis-propagating effects of the hemostatic system are at least in part mediated by protection of tumor cells from NK cell reactivity. While mouse studies on pharmacological interference in metastasis have raised more questions than answers, the diligent work of Palumbo et al. [19] helped to at least partly dissect platelet- versus coagulation factor-dependent mechanisms. Clearly, a thorough elucidation of mechanisms exclusively mediated by platelets is not fully achievable in mouse models and rather, to our opinion, requires in vitro and ex vivo studies, preferably performed with human cells, in which suitable experimental conditions can be applied to define specific molecular mechanisms.

\section{Tumor-Platelet-NK Cell Interaction in the Human System}

Tumor cell-platelet-NK cell interactions resulting in altered effector functions of NK cells may be mediated by platelet-derived soluble factors and direct platelet-NK cell interaction. Available results provide evidence that both mechanisms are involved.

\section{Effects of Platelet-Derived Soluble Factors}

In 1986, Skov et al. [20] demonstrated that supernatants from thrombin-stimulated platelets (but not thrombin itself) significantly reduced lysis of K562 cells by human NK cells. The responsible mechanism was not determined in this early study. However, NK cells have been reported to express the thrombin receptor [21], and some years later, Naldini and Carney [22] reported that throm- 
bin, which can also be released by platelets, increased cytotoxicity of peripheral blood mononuclear cells and the NK cell line NK3.3, while effector functions of lymphokine-activated killer cells were inhibited. While the reasons for the discrepancy of their results with regard to the investigated cell types, but also to the results of Skov et al. [20], remain unclear, these data can be interpreted as evidence that platelet-derived soluble factors may mediate effects on natural cytotoxicity in a context-dependent manner [22]. In a subsequent study, Naldini et al. [23] analyzed the effect of platelet gels (obtained by treating platelet concentrates with autologous thrombin), which are being evaluated as means to improve tissue healing and regeneration, on cytokine production of peripheral blood mononuclear cells. The presence of these platelet preparations enhanced the lipopolysaccharide-induced release of various cytokines such as IL-1 $\beta$, IL- 6 and IL-8, but significantly reduced the release of interferon (IFN)$\gamma$ and IL-12. While it seems likely that the observed effects were due to soluble factors released by the platelets, it should be noted that the experimental setting of these analyses does not rule out effects of platelet membranebound immunoregulatory molecules.

We recently demonstrated that platelet-derived soluble factors released upon platelet activation with classical agonists like thrombin or collagen, but also upon interaction of platelets with tumor cells, diminish NK cell cytotoxicity and IFN- $\gamma$ production in response to tumor targets. The platelet agonists themselves had no relevant influence on the reactivity of the polyclonal NK cells employed in our experimental setting. The inhibitory effect of the platelet releasate was at least partially mediated by downregulation of NKG2D on the NK cells. NKG2D is an activating NK cell receptor and plays a prototypical role in 'induced self' recognition upon binding to its stress-induced ligands (NKG2DL) [for a review, see ref. 1]. The reduction in NKG2D expression observed in our experiments was, at least to a great part, due to plateletderived transforming growth factor (TGF)- $\beta$, as a TGF$\beta$-neutralizing antibody restored NKG2D expression and largely prevented impairment of NK cell reactivity. Our finding that the inhibitory effect of platelet releasate on NK cell anti-tumor reactivity was dependent on NKG2DL expression on the target cells further confirmed the relevance of NKG2D-mediated target recognition in our model [24]. As platelets are known to corelease both TGF$\beta$ and its most potent activator thrombospondin 1 [25], these data indicate that platelets, upon interaction with (metastasizing) tumor cells, may release factors which enable immune evasion of tumor cells from NK cell im- mune surveillance by disturbing 'induced self' recognition. Of note, thrombospondin was further reported to modulate feeder cell-induced NK cell proliferation in vitro via activation of TGF- $\beta$, and its effect was dependent on the time point of administration [26]. It should be noted that beyond TGF- $\beta$, platelets have been found to release a multitude of other growth factors with potential influence on NK cell function. An obvious example is PDGF, which, while not studied directly in a model investigating the trilateral interaction of platelets, tumor cells and NK cells, has been reported to modulate NK cell reactivity. Gersuk et al. [27] reported that preincubation of NK cells with PDGF reduced the binding of NK cells to K562 target cells as well as direct target cell lysis in a time- and dose-dependent manner. Interestingly, exposure of PDGF-treated NK cells to IFN- $\alpha$ or IL-2 restored NK cell reactivity, and PDGF did not impair antibodydependent cellular cytotoxicity or cytotoxicity of IL-2/ IFN- $\alpha$-pretreated NK cells. These findings add further evidence to our notion that the effects of platelet-derived soluble factors on NK cell reactivity may depend on the cellular context and NK cell activity levels. Moreover, different isoforms of PDGF (all three reportedly secreted by platelets) were found to inhibit NK cells with different potency. Pretreatment of NK cells with PDGF-AA resulted in a weaker inhibition compared to PBGF-AB and PDGF-BB [27]. In contrast to the inhibitory effect of PDGF, insulin-like growth factor (IGF) I, which can also be released by platelets, was reported to enhance lytic activity of NK cells, and IGF I levels in growth hormonedeficient patients correlated with NK cell reactivity [28]. Further evidence suggests that VEGF, which is also largely secreted by platelets, may promote adhesion of NK cells to tumor vessels by increasing the expression of intercellular adhesion molecule 1 (ICAM-1), vascular cell adhesion molecule 1 (VCAM-1) and E-selectin, whereas basic fibroblast growth factor (bFGF) counteracts NK cell adhesion to endothelial cells [29].

Platelets further contain and release various chemokines governing migration of immune cells to plateletactivating sites, which includes neoangiogenic vascular endothelium [30]. Among others, platelet factor 4, now termed 'CXCL4', potently attracts immune effector cells to sites of platelet degranulation/inflammation. Moreover, Marti et al. [31] reported that platelet factor 4 stimulates NK cells to produce and release functional IL-8 via activation of phosphoinositide-3 kinase signaling. Thereby, platelet-stimulated NK cells may contribute to chemoattraction and most likely also to modulation of neutrophil reactivity. The cognate receptors of further plate- 
let-derived chemokines including CCL3, CCL5 and CXCL12 are expressed on NK cells [for a review, see ref. $32,33]$, but their functional role in platelet-NK cell interaction has not yet been studied. Moreover, the exact composition of platelet releasate with regard to factors influencing NK cell reactivity remains to be defined and may comprise yet unknown molecules that unexpectedly modulate NK cell reactivity [34].

What can be concluded from the available data is that (1) platelet releasate contains both stimulatory and inhibitory factors, and their effect on NK cell reactivity is likely to depend on their relative rather than absolute concentrations as well as on the cellular context and NK cell activation state. (2) Releasates may vary inter-individually and, more importantly, between healthy persons and cancer patients. (3) The factors released by platelets may differ dependent on the mechanisms leading to secretion, as highlighted recently by data on the consequences of triggering different thrombin receptors (PAR1 vs. PAR4) for angiogenesis [35]. Clearly, more work is needed to elucidate the complexity of the factors contained in platelet releasate and their functional interplay, not only with regard to modulation of NK cell anti-tumor reactivity, but to their influence on cellular reactions overall.

\section{Effects of Immunoregulatory Molecules Expressed on} the Platelet Surface

Platelets express various membrane-bound molecules by which they may modulate NK cell reactivity. The important role of platelet-expressed adhesion molecules in various physiological and pathophysiological conditions is well defined [for excellent reviews, see ref. 4, 36]. Neoangiogenesis is a hallmark of cancer, and platelets preferentially adhere to neoangiogenic vessels [37]. Immobilized and thus activated platelets express P-selectin, which supports NK cell tethering and rolling via ligation of P-selectin glycoprotein ligand 1 , leading to $\beta_{2}$-integrin-dependent adhesion and infiltration of $\mathrm{NK}$ cells into their sites of action [36]. Of note, the highly cytolytic $\mathrm{CD} 56^{\mathrm{dim}} \mathrm{CD} 16^{+} \mathrm{NK}$ cell subset expresses PEN5, a modified form of P-selectin glycoprotein ligand 1 which can bind to L-selectin, and PEN5 expression is associated with upregulation of KIR [38, 39]. It is tempting to speculate that other NK cell subsets expressing P-selectin ligands with preferential binding to activated platelets may exist and may specifically be involved in NK cell-platelet interaction; however, this has not been studied yet.

Apart from adhesion molecules, platelets express various other immunoregulatory molecules, by which they may influence NK cell anti-tumor reactivity following adhesion/aggregation to (metastasizing) tumor cells. For example, platelets express various members of the TNF superfamily. Among others, both CD40 and its ligand (CD40L) are expressed on platelets and influence cytotoxicity and cytokine production of $\mathrm{T}$ cells as well as proliferation and antibody production of $\mathrm{B}$ cells [for a review, see ref. 36]. While the role of the CD40/CD40L molecule system in the interaction of platelets and NK cells has not yet been studied, NK cells have been shown to upregulate CD40L upon activation and to mediate enhanced cytotoxicity upon CD40L triggering [40]. Moreover, TWEAK, LIGHT, OX40 ligand and other TNF family members are expressed on or released by platelets, while their counterparts can be expressed on NK cells [41-45]. The functional role of these molecule systems in plateletNK cell interaction has not yet been investigated. We found that the TNF family member glucocorticoid-induced TNF receptor-related (GITR) ligand is upregulated during megakaryopoiesis resulting in substantial expression by platelets. As GITR-GITR ligand (GITRL) interaction transduces bidirectional signals, i.e. not only into GITR- but also into GITRL-bearing cells [46], we studied whether GITRL signaling altered platelet activation. Analysis of P-selectin and release of TGF- $\beta$ as surrogate markers for activation revealed no influence of GITRL signaling in platelets. However, we found evidence that platelet-expressed GITRL may affect NK cell anti-tumor immunity upon platelet-tumor interaction. There is ample evidence that both disseminating tumor cells and leukemic blasts do not travel through the blood alone, but surround themselves by coating platelets $[47,48]$. Mechanistic hypotheses proposed that tumor cells may 'hide' behind platelets, thereby preventing access of immune cells. Our in vitro analyses revealed that tumor cells in fact rapidly get coated by platelets, which confers a seemingly GITRL-positive phenotype to constitutively GITRL-negative cancer cells. Since we recently identified GITR as an inhibitory NK cell receptor which, in contrast to its stimulatory role in T cells, may contribute to the escape of GITRL-expressing targets from immune surveillance of NK cells $[49,50]$, we studied whether and how platelet-derived GITRL influenced NK cell reactivity. 'GITRL pseudo expression' on tumor cells following coating by platelets substantially reduced cytotoxicity and IFN- $\gamma$ production of allogenic NK cells. The reduced NK cell reactivity was not due to induction of apoptosis via GITR and could partially be restored by a blocking GITR antibody, which confirmed that transfer of GITRL to tumor cells by coating platelets inhibits NK cell reactivity [51] [manuscript in prep.]. 
In addition, we obtained evidence that platelet coating enables tumor cells to evade NK cell immune surveillance by disturbing the recognition of 'missing self'. Platelets express high levels of MHC class I and get activated upon coating of cancer cells. Consequently, the platelet membrane and platelet microparticles adhere to and may even integrate into the tumor cell membrane, thereby transferring MHC class I. Thus, platelets can confer an immunophenotype of false pretenses - or 'pseudo self' - to tumor cells. This may allow the latter to downregulate their own MHC class I in order to escape T-cell immunity while being protected from autologous NK cells by platelet-derived MHC class I, e.g., when traveling through the blood. In line with this hypothesis, we found that the impaired lysis of platelet-coated tumor cells by NK cells of the platelet donor could be restored in great part by blockade of MHC class I. Of note, blocking MHC class I on uncoated tumor cells had no effect in our in vitro model, regardless whether the employed tumor targets (allogenic to the NK cell/platelet donor) expressed MHC class I or were MHC class I negative [52] [manuscript in prep.].

Together, the data from our coating experiments indicate that platelets may enable a molecular mimicry of tumor cells and can protect malignant cells from NK cell anti-tumor immunity by conferring 'pseudo expression' of immunoregulatory molecules including 'pseudo self'. Our findings in the human system seem to contrast with those obtained in mice by Nieswandt et al. [6], who also observed a clear inhibition of YAC1 killing by splenic effector cells upon addition of platelets. As reduced lysis was observed with platelets from different allogenic mouse strains and MHC class I low/negative $\beta_{2}$-microglobulin-deficient mice, these investigators concluded that MHC class-I-independent mechanisms were responsible for tumor protection. However, since platelets were added to NK cell-tumor cell cocultures instead of coating tumor cells prior to blocking MHC class I as in our experiments, soluble factors (e.g., TGF$\beta)$, which are released upon platelet aggregation, certainly played a role and may have interfered with the detection of effects of MHC class I. Indeed, supernatants of platelets also mediated NK cell inhibitory effects in their analyses, and inhibition of aggregation prevented the reduction of NK cell reactivity. Furthermore, effects of other platelet-expressed and potentially immunoinhibitory molecules were not excluded in these experiments. As NK cell reactivity is governed by a balance of multiple activating and inhibitory signals, the results of Nieswandt et al. [6] do not contradict our findings but rather support our notion that platelets may influence NK cell anti-tumor reactivity by a whole variety of mechanisms which comprise but are not limited to disturbing the principles of the 'missing self' and 'induced self'.

\section{Conclusion and Outlook}

NK cells play an important role in tumor immune surveillance. Their reactivity is not only governed by signals mediated by inhibitory and activating ligands expressed on their target cells, but also by various other cells of the hematopoietic system [2]. Accumulating evidence demonstrates that platelets, beyond being a principal element in thrombosis and hemostasis, may facilitate tumor immune escape and metastasis. While platelets may influence many components of anti-tumor immunity, the available data clearly indicate that this is, at least to a great part, mediated by modulation of NK cell reactivity. It should be noted that, vice versa, lymphocyte-derived factors and cytokines like IFN- $\gamma$, IL- 2 and many others may influence platelet functions [for a review, see ref. 36]. For interpretation of the available data, one should also bear in mind that in mouse models of experimental metastasis, large numbers of tumor cells are intravenously injected, which does not reflect the true situation in patients, where metastasis is a rare event with few tumor cells entering the blood stream. The crosstalk of platelets, tumor cells and NK cells in tumor development and metastasis constitutes a complex mechanism that is influenced by a multitude of different factors which we are just beginning to understand. It is difficult to envisage valid studies assessing the effect of anti-platelet strategies on metastasis in humans, and the reported primary preventive effect of aspirin in colorectal cancer is mediated by direct effects on tumor cells rather than by effects on platelets [53]. Similarly, low molecular weight heparin, which was reported to prolong survival in cancer patients with venous thromboembolism, does not directly target platelets [54]. In mouse models, inhibitors of aggregation, such as the classical platelet antagonists aspirin, ticlopidin and $\mathrm{PGI}_{2}$, had no relevant effect on metastasis $[11,12]$. Such platelet antagonists inhibit platelet aggregation and activation, thereby reducing the release of soluble NK cell modulatory factors [55], but they fail to effectively inhibit adhesion of platelets to tumor cells, which allows for effects of platelet membrane-bound molecules [8]. Clearly, platelets influence NK cell reactivity on several different levels, and both soluble and membrane-bound factors 
may be required for NK cell evasion of tumor cells in vivo. Novel agents aiming to reinforce NK cell reactivity by modulating platelet-NK cell interaction would thus need to inhibit both platelet aggregation and adhesion to tumor cells. Such agents would ideally not confer a bleeding risk and could then be utilized to prevent unintended tumor spread in a neoadjuvant treatment setting, thereby helping to further improve long-term outcomes in patients with tumors typically treated preoperatively (e.g. colorectal cancer and sarcoma). While much further work both in vivo and in vitro as well as in the murine and the human system is required to delineate the underlying mechanisms, a better understanding of the potentially fatal 'ménage a trois' of tumor cells, platelets and
NK cells clearly holds promise to improve future immunotherapeutic approaches in cancer therapy, especially those relying on sufficient NK cell reactivity.

\section{Acknowledgements}

This work was supported by grants from the German Research Foundation (SFB685, project A7) and the Max Eder Stiftung, Deutsche Krebshilfe.

\section{Disclosure Statement}

The authors declare no conflicts of interest.

\section{References}

$>1$ Lanier LL: NK cell recognition. Annu Rev $>11$ Immunol 2005;23:225-274.

-2 Vivier E, Tomasello E, Baratin M, Walzer T, Ugolini S: Functions of natural killer cells. Nat Immunol 2008;9:503-510.

3 Billroth T: Lectures on Surgical Pathology and Therapeutics: A Handbook for Students and Practitioners. London, New Sydenham Society, 1878.

4 Erpenbeck L, Schon MP: Deadly allies: the fatal interplay between platelets and metastasizing cancer cells. Blood 2010;115:34273436.

5 Gasic GJ, Gasic TB, Stewart CC: Antimetastatic effects associated with platelet reduction. Proc Natl Acad Sci USA 1968;61:46-52.

-6 Nieswandt B, Hafner M, Echtenacher B, Mannel DN: Lysis of tumor cells by natural killer cells in mice is impeded by platelets. Cancer Res 1999;59:1295-1300.

-7 Gasic GJ, Gasic TB, Galanti N, Johnson T, Murphy S: Platelet-tumor-cell interactions in mice. The role of platelets in the spread of malignant disease. Int J Cancer 1973;11:704718 .

8 Nierodzik ML, Karpatkin S: Thrombin induces tumor growth, metastasis, and angiogenesis: evidence for a thrombin-regulated dormant tumor phenotype. Cancer Cell 2006;10:355-362.

-9 Rickles FR, Falanga A: Molecular basis for the relationship between thrombosis and cancer. Thromb Res 2001;102:V215-V224.

10 Pearlstein E, Salk PL, Yogeeswaran G, Karpatkin S: Correlation between spontaneous metastatic potential, platelet-aggregating activity of cell surface extracts, and cell surface sialylation in 10 metastatic-variant derivatives of a rat renal sarcoma cell line. Proc Natl Acad Sci USA 1980;77:4336-4339.
Karpatkin S, Pearlstein E, Ambrogio C, Coller BS: Role of adhesive proteins in platelet tumor interaction in vitro and metastasis formation in vivo. J Clin Invest 1988;81: 1012-1019.

12 Karpatkin S, Ambrogio C, Pearlstein E: Lack of effect of in vivo prostacyclin on the development of pulmonary metastases in mice following intravenous injection of CT26 colon carcinoma, Lewis lung carcinoma, or B16 amelanotic melanoma cells. Cancer Res 1984;44:3880-3883.

13 Gorelik E, Bere WW, Herberman RB: Role of NK cells in the antimetastatic effect of anticoagulant drugs. Int J Cancer 1984;33:87-94.

14 Jin DK, Shido K, Kopp HG, Petit I, Shmelkov SV, Young LM, Hooper AT, Amano H, Avecilla ST, Heissig B, Hattori K, Zhang F, Hicklin DJ, Wu Y, Zhu Z, Dunn A, Salari H, Werb Z, Hackett NR, Crystal RG, Lyden D, Rafii S: Cytokine-mediated deployment of SDF-1 induces revascularization through recruitment of CXCR4+ hemangiocytes. Nat Med 2006;12:557-567.

15 Gurney AL, de Sauvage FJ: Dissection of c$\mathrm{Mpl}$ and thrombopoietin function: studies of knockout mice and receptor signal transduction. Stem Cells 1996;14(suppl 1):116-123.

-16 Palumbo JS, Talmage KE, Massari JV, La Jeunesse CM, Flick MJ, Kombrinck KW, Jirouskova M, Degen JL: Platelets and fibrin(ogen) increase metastatic potential by impeding natural killer cell-mediated elimination of tumor cells. Blood 2005;105:178-185.

17 Palumbo JS, Talmage KE, Massari JV, La Jeunesse CM, Flick MJ, Kombrinck KW, Hu Z, Barney KA, Degen JL: Tumor cell-associated tissue factor and circulating hemostatic factors cooperate to increase metastatic potential through natural killer cell-dependent and -independent mechanisms. Blood 2007; 110:133-141.
18 Zheng S, Shen J, Jiao Y, Liu Y, Zhang C, Wei $\mathrm{M}$, Hao S, Zeng X: Platelets and fibrinogen facilitate each other in protecting tumor cells from natural killer cytotoxicity. Cancer Sci 2009;100:859-865.

19 Palumbo JS, Barney KA, Blevins EA, Shaw MA, Mishra A, Flick MJ, Kombrinck KW, Talmage KE, Souri M, Ichinose A, Degen JL: Factor XIII transglutaminase supports hematogenous tumor cell metastasis through a mechanism dependent on natural killer cell function. J Thromb Haemost 2008;6:812819.

20 Skov MP, Hokland P, Hokland M: Secretory products from thrombin-stimulated human platelets exert an inhibitory effect on NK-cytotoxic activity. Acta Pathol Microbiol Immunol Scand C 1986;94:193-200.

21 Howells GL, Macey M, Murrell AM, Green AR, Curtis MA, Stone SR: Thrombin receptor expression and function in large granular lymphocyte proliferative disorders. Br J Haematol 1994;88:383-388.

22 Naldini A, Carney DH: Thrombin modulation of natural killer activity in human peripheral lymphocytes. Cell Immunol 1996; 172:35-42.

23 Naldini A, Morena E, Fimiani M, Campoccia G, Fossombroni V, Carraro F: The effects of autologous platelet gel on inflammatory cytokine response in human peripheral blood mononuclear cells. Platelets 2008;19: 268-274.

- 24 Kopp HG, Placke T, Salih HR: Platelet-derived transforming growth factor-beta down-regulates NKG2D thereby inhibiting natural killer cell antitumor reactivity. Cancer Res 2009;69:7775-7783. 
25 Ahamed J, Janczak CA, Wittkowski KM, Coller BS: In vitro and in vivo evidence that thrombospondin-1 (TSP-1) contributes to stirring- and shear-dependent activation of platelet-derived TGF-beta1. PLoS One 2009; 4:e6608.

-26 Pierson BA, Gupta K, Hu WS, Miller JS: Human natural killer cell expansion is regulated by thrombospondin-mediated activation of transforming growth factor-beta 1 and independent accessory cell-derived contact and soluble factors. Blood 1996;87:180-189.

27 Gersuk GM, Westermark B, Mohabeer AJ, Challita PM, Pattamakom S, Pattengale PK: Inhibition of human natural killer cell activity by platelet-derived growth factor (PDGF). 3. Membrane binding studies and differential biological effect of recombinant PDGF isoforms. Scand J Immunol 1991;33:521-532.

- 28 Auernhammer CJ, Feldmeier H, Nass R, Pachmann K, Strasburger CJ: Insulin-like growth factor I is an independent coregulatory modulator of natural killer (NK) cell activity. Endocrinology 1996;137:5332-5336.

-29 Melder RJ, Koenig GC, Witwer BP, Safabakhsh N, Munn LL, Jain RK: During angiogenesis, vascular endothelial growth factor and basic fibroblast growth factor regulate natural killer cell adhesion to tumor endothelium. Nat Med 1996;2:992-997.

- 30 Wiesner T, Bugl S, Mayer F, Hartmann JT, Kopp HG: Differential changes in platelet VEGF, Tsp, CXCL12, and CXCL4 in patients with metastatic cancer. Clin Exp Metastasis 2010;27:141-149.

- 31 Marti F, Bertran E, Llucia M, Villen E, Peiro M, Garcia J, Rueda F: Platelet factor 4 induces human natural killer cells to synthesize and release interleukin-8. J Leukoc Biol 2002;72:590-597.

32 Robertson MJ: Role of chemokines in the biology of natural killer cells. J Leukoc Biol 2002;71:173-183.

33 Flad HD, Brandt E: Platelet-derived chemokines: pathophysiology and therapeutic aspects. Cell Mol Life Sci 2010;67:2363-2386.

- 34 Klein-Struckmeier A, Knuver-Hopf J, Mohr $\mathrm{H}$ : Modulation of natural and interleukin-2induced tumour-cytolytic activities by the members of a protein family related to betathromboglobulin. Cancer Immunol Immunother 1991;34:175-180.
Italiano JE Jr, Richardson JL, Patel-Hett S, Battinelli E, Zaslavsky A, Short S, Ryeom S, Folkman J, Klement GL: Angiogenesis is regulated by a novel mechanism: pro- and antiangiogenic proteins are organized into separate platelet alpha granules and differentially released. Blood 2008;111:1227-1233.

36 Li N: Platelet-lymphocyte cross-talk. J Leukoc Biol 2008;83:1069-1078.

37 Kisucka J, Butterfield CE, Duda DG, Eichenberger SC, Saffaripour S, Ware J, Ruggeri ZM, Jain RK, Folkman J, Wagner DD: Platelets and platelet adhesion support angiogenesis while preventing excessive hemorrhage. Proc Natl Acad Sci USA 2006;103:855-860.

- 38 Andre P, Spertini O, Guia S, Rihet P, DignatGeorge F, Brailly H, Sampol J, Anderson PJ, Vivier E: Modification of P-selectin glycoprotein ligand-1 with a natural killer cell-restricted sulfated lactosamine creates an alternate ligand for L-selectin. Proc Natl Acad Sci USA 2000;97:3400-3405.

39 Vivier E, Sorrell JM, Ackerly M, Robertson MJ, Rasmussen RA, Levine H, Anderson P: Developmental regulation of a mucinlike glycoprotein selectively expressed on natural killer cells. J Exp Med 1993;178:2023-2033.

40 Carbone E, Ruggiero G, Terrazzano G, Palomba C, Manzo C, Fontana S, Spits H, Karre $\mathrm{K}$, Zappacosta S: A new mechanism of NK cell cytotoxicity activation: the CD40-CD40 ligand interaction. J Exp Med 1997;185: 2053-2060

41 Meyer T, Amaya M, Desai H, Robles-Carrillo L, Hatfield M, Francis JL, Amirkhosravi A: Human platelets contain and release TWEAK. Platelets 2010;21:571-574.

42 Otterdal K, Smith C, Oie E, Pedersen TM, Yndestad A, Stang E, Endresen K, Solum NO, Aukrust P, Damas JK: Platelet-derived LIGHT induces inflammatory responses in endothelial cells and monocytes. Blood 2006;108:928-935.

43 Liu DM, Yan JC, Wang CP, Chen GH, Ding S, Liu PJ, Du RZ: The clinical implications of increased OX40 ligand expression in patients with acute coronary syndrome. Clin Chim Acta 2008;397:22-26.

44 Dybkaer K, Iqbal J, Zhou G, Geng H, Xiao L, Schmitz A, d'Amore F, Chan WC: Genome wide transcriptional analysis of resting and IL2 activated human natural killer cells: gene expression signatures indicative of novel molecular signaling pathways. BMC Genomics 2007;8:230.

-45 Maecker H, Varfolomeev E, Kischkel F, Lawrence D, LeBlanc H, Lee W, Hurst S, Danilenko D, Li J, Filvaroff E, Yang B, Daniel D, Ashkenazi A: TWEAK attenuates the transition from innate to adaptive immunity. Cell 2005; 123:931-944.
46 Placke T, Kopp HG, Salih HR: Glucocorticoid-induced TNFR-related (GITR) protein and its ligand in antitumor immunity: functional role and therapeutic modulation. Clin Dev Immunol 2010;2010:239083.

47 Borsig L, Wong R, Feramisco J, Nadeau DR, Varki NM, Varki A: Heparin and cancer revisited: mechanistic connections involving platelets, P-selectin, carcinoma mucins, and tumor metastasis. Proc Natl Acad Sci USA 2001;98:3352-3357.

- 48 Betz SA, Foucar K, Head DR, Chen IM, Willman CL: False-positive flow cytometric platelet glycoprotein IIb/IIIa expression in myeloid leukemias secondary to platelet adherence to blasts. Blood 1992;79:2399-2403.

- 49 Baltz KM, Krusch M, Bringmann A, Brossart P, Mayer F, Kloss M, Baessler T, Kumbier I, Peterfi A, Kupka S, Kroeber S, Menzel D, Radsak MP, Rammensee HG, Salih HR: Cancer immunoediting by GITR (glucocorticoid-induced TNF-related protein) ligand in humans: NK cell/tumor cell interactions. FASEB J 2007;21:2442-2454.

50 Baltz KM, Krusch M, Baessler T, Schmiedel BJ, Bringmann A, Brossart P, Salih HR: Neutralization of tumor-derived soluble glucocorticoid-induced TNFR-related protein ligand increases NK cell anti-tumor reactivity. Blood 2008;112:3735-3743.

51 Placke T, Kanz L, Salih HR, Kopp HG: Pseudoexpression of glucocorticoid-induced TNF-related (GITR) ligand upon coating of cancer cells by platelets impairs NK cell antitumor reactivity. Blood 2010;116:3193.

52 Placke T, Kopp HG, Schaller M, Jung G, Kanz L, Salih HR: Transfer of MHC class I by coating platelets enables tumor cells to evade NK cell immune surveillance by conferring a 'pseudo self' phenotype. Blood 2010;116:583.

53 Das D, Arber N, Jankowski JA: Chemoprevention of colorectal cancer. Digestion 2007; 76:51-67.

54 Lee AY, Rickles FR, Julian JA, Gent M, Baker RI, Bowden C, Kakkar AK, Prins M, Levine MN: Randomized comparison of low molecular weight heparin and coumarin derivatives on the survival of patients with cancer and venous thromboembolism. J Clin Oncol 2005;23:2123-2129.

55 Coppinger JA, O'Connor R, Wynne K, Flanagan M, Sullivan M, Maguire PB, Fitzgerald DJ, Cagney G: Moderation of the platelet releasate response by aspirin. Blood 2007; 109:4786-4792. 